\title{
Is there Optimal Behavioral Treatment Intensity for Dysphagia?
}

Kaipa $\mathrm{R}^{1 *}$, Thomas $\mathrm{RM}^{2}$

${ }^{1}$ Department of Communication Sciences and Disorders, Oklahoma State University, Stillwater, OK, USA.

${ }^{2}$ Department of Psychology, Oklahoma State University, Stillwater, OK, USA.

\begin{abstract}
Purpose: Determining optimal intensity of behavioral treatment for dysphagia has been one of the most debated topics. The purpose of the current study was to systematically review the studies that offered evidence for behavioral treatment intensity for dysphagia.

Methods: A systematic search was conducted in six scientific databases using 16 different combinations of keywords, in addition to manually searching different journals for relevant articles. A total of 50,192 articles were obtained from the initial searches. After rigorous screening, only five articles met the inclusion criteria. All studies were evaluated for their methodological quality, evidence level, and for pertinent information regarding treatment intensity.

Results: The results of the systematic review revealed that only one study presented sufficient information that allowed the authors to determine cumulative treatment intensity for dysphagia. The research designs employed by the studies ranged from randomized-controlled design to non-experimental retrospective design. Appraisal of the methodological quality revealed that only two articles had moderate internal validity, and the remaining articles demonstrated poor internal validity. Conclusion: The findings suggest that there is limited evidence regarding optimal behavioral treatment intensity for dysphagia, and there is a need for well-controlled group design studies to expand on this line of research.
\end{abstract}

Keywords: Treatment Intensity; Dysphagia; Randomized-Controlled Trials; Systematic Review; Methodological Quality.

\section{*Corresponding Author:}

Dr. Ramesh Kaipa,

Department of Communication Sciences and Disorders, Oklahoma State University, Stillwater, OK 74074, USA.

Tel: +1-405-744-7956

Fax: +1-405-364-2760

E-mail: ramesh.kaipa@okstate.edu

Received: September 16, 2015

Accepted: October 28, 2015

Published: October 29, 2015

Citation: Kaipa R, Thomas RM (2015) Is there Optimal Behavioral Treatment Intensity for Dysphagia?. Int J Clin Exp Otolaryngol. 01(2), 1018. doi: http://dx.doi.org/10.19070/2572-732X-150002

Copyright: Kaipa $\mathbf{R}^{\odot}$ 2015. This is an open-access article distributed under the terms of the Creative Commons Attribution License, which permits unrestricted use, distribution and reproduction in any medium, provided the original author and source are credited.

\section{Introduction}

Difficulty in swallowing referred to as dysphagia affects 1 out of every 25 individuals annually [1]. Individuals identified with dysphagia not only include adults in acute and chronic care facilities [2] but also pediatric population. Cichero and Altman [3] estimated the annual hospital cost associated with dysphagia to be USD 547 million. This suggests an increased economic burden on the healthcare system. The strong relationship between swallowing function, nutritional status and health outcome suggests the significance of appropriate dysphagia management [2]. With the ever-increasing cost associated with dysphagia management, the importance of evidence-based treatment in dysphagia cannot be underestimated. Speech-language pathologists (SLPs) are health care professionals who play a major role not only in assessment and diagnosis of dysphagia, but also in treatment [4]. Despite significant advancements in dysphagia intervention over the past few years [5], a relevant question that remains to be answered is how much treatment for dysphagia is considered to be sufficient. Studying optimal treatment intensity is fundamental to practice of speech-language pathology [6], and SLPs have frequently debated the optimal intervention intensity for dysphagia with limited success.

Multiple approaches have been employed by previous studies to determine treatment intensity in speech-language pathology. For example, Bhogal, Teasell and Speechley [7] estimated treatment intensity by counting the number of hours of therapy per week. Whereas, Brandel and Loeb [8] determined treatment intensity by counting the number and duration of sessions per week. Treatment intensity cannot be determined merely by counting the number of hours or number of intervention sessions, as different factors needed to be accounted for while determining treatment intensity. Warren, Fey, \& Yoder [9] recommended a holistic model for determining treatment intensity in speech-language pathology. Warren et al. suggested five variables to be taken into account to determine treatment intensity. The five variables are dose, dose form, dose frequency, total intervention duration, and cumulative intervention intensity. Dose form refers to the activity that contain the active ingredients of intervention. For example, slowing down the patient's rate of feeding (active ingredient) during dysphagia therapy. The number of times an active ingredient is delivered within an intervention session is referred to as the dose (e.g., 
100 swallowing trials during a 60-minute therapy session). It is important to quantify the session duration to determine dose. Dose frequency can be estimated based on the number of intervention sessions that are provided per unit time. (e.g., $3 \times$ day, $6 \times$ week, $8 \times$ month). The total time period of intervention is referred to as the total intervention duration (e.g., 8 weeks, 6 months). Finally, the product of dose, dose frequency, and total intervention duration yields cumulative intervention intensity.

A handful of studies in the past have investigated treatment outcomes in dysphagia as a function of treatment intensity [e.g.,10, 11]. But the results have been equivocal in terms of optimal treatment intensity required for dysphagia intervention. For example, McCullough et al. [10] found that patients with dysphagia treated using the Mendelsohn maneuver 10 times a week (approx.) for two weeks demonstrated considerable improvement in their swallowing function. On the other hand, Bakhtiyari et al. [11] found that when patients with dysphagia were treated using traditional behavioral intervention approaches for 3-times a week for a total period of 3-months, the patients showed considerable improvement.

Even though determining optimal treatment intensity for dysphagia intervention is challenging, it is essential to pursue this line of research to promote evidence-based practice (EBP) in management of dysphagia. EBP aims at integrating clinical expertise, best evidence and client/patient/caregiver perspectives [12]. Currently, the optimal treatment intensity required for successfully treating patients with dysphagia remains unknown. So, the current study aimed at systematically reviewing past studies that investigated treatment intensity in patients with dysphagia, and also to evaluate the methodological quality of these studies using a validated assessment tool. To our knowledge this is the first study to systematically review previous literature on treatment intensity in behavioral management of dysphagia. The current study adopted the model recommended by Warren et al. [9] to investigate existing evidence regarding behavioral treatment intensity for dysphagia.

\section{Method}

To maintain transparency and replicability, the current study followed the guidelines of Preferred Reporting Items for Systematic reviews and Meta-Analyses (PRISMA) [13]. The PRISMA flow chart is depicted in Figure 1 (adapted from [13]).

\section{Identification}

Search process: A systematic literature search was conducted by the second author using the following databases: Web of Science, MEDLINE, ProQuest, PubMed, Science Direct and Google Scholar. In addition, the first and second authors manually searched for relevant articles in the appropriate journal collections of the authors' University library website. The search terms comprised of 16 different combinations of keywords. Each of the 16 combinations of keywords was entered into each of the abovementioned databases to conduct the literature search. The initial exhaustive search yielded a total number of 50,192 articles including the one article that was obtained through manual search. Only one article was obtained through manual search. The details of 16 combinations of keywords are mentioned in appendix.

\section{Screening}

Inclusion criteria: Studies involving human participants published prior to August 2015 in peer-reviewed journals were included in this review. Longitudinal studies with and without control groups, cross-sectional studies with and without control groups, single case-experimental design studies (SCEDs), one-shot prepost designs, and randomized controlled studies published in

Figure 1. Schematic representation of the search process (adapted from Preferred Reporting Items for Systematic reviews and Meta-Analyses (PRISMA) [13]). RCTs = randomized-controlled trials.
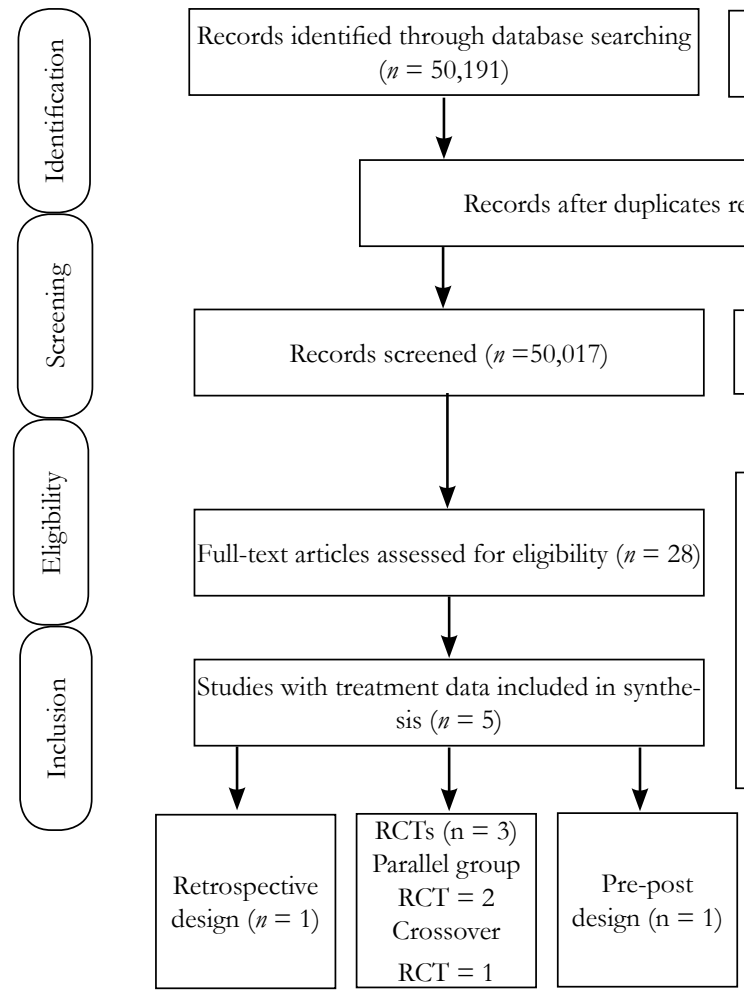

Additional records identified through other sources $(n=1)$

Full-text articles excluded, due to not mee ing criteria:

1. Review articles

2. Commentaries/opinion articles

3. Did not focus on behavioral treatment

4. Survey articles

5. Tutorials

6. Did not provide adequate information about treatment intensity 
English were considered for this review. Only studies that offered evidence about treatment intensity in behavioral management of dysphagia (irrespective of the etiology) were included in the current review.

Exclusion criteria: Studies that focused on other types of intervention for dysphagia (e.g., transcranial magnetic stimulation, surgery) were excluded from this review. Opinion articles, letters to editors, commentaries and review articles were not considered for this study.

A total of 175 duplicate articles obtained during the search process were excluded from 50,192 articles. The remaining 50,017 articles were screened by applying inclusion and exclusion criteria to their title and abstracts. This resulted in exclusion of 49,989 articles. The remaining 28 full-text articles were assessed for eligibility.

\section{Eligibility}

The second author read the 28 articles thoroughly and eliminated another 23 articles. The full-length articles were eliminated due to following reasons: (1) commentaries or opinion articles on dysphagia treatment, (2) tutorials, (3) systematic reviews, (4) did not focus on behavioral treatment of dysphagia, (5) did not provide adequate information on behavioral treatment intensity of dysphagia, and (5) were survey articles. Finally, only 5 articles met the inclusion criteria and were included in the review.

\section{Methodological assessment of the eligible studies}

The methodological quality of the five studies was evaluated using a standardized tool called PEDro-P scale [14]. The PEDro-P scale was adapted from the original PEDro scale [15].The PEDro-P scale was designed to evaluate the external and internal validity of randomized controlled trials (RCTs) and non-randomized controlled trials (NRCTs). The scale takes 11 criteria into consideration in determining the external and internal validity of a study. The first criterion on the scale ("eligibility criteria") is related to external validity. Criteria 2-9 evaluates the internal validity of a study by considering a number of variables such as random allocation, concealment of allocation, similarity of the groups at baseline, blinding of subjects and therapists, and so forth. The last two criteria evaluate the interpretability by checking for between-group statistical comparisons and if both point estimates and variability measures are reported. Each criterion on the scale is awarded a score of either " 1 " (if the study meets that criterion) or " 0 " (if the study does not meet that criterion). Only criteria 2-11 are scored, so the maximum score a paper can achieve on the PEDro-P scale is " 10 " [14].

It is important to note that PEDro-P scale is exclusively used for rating the methodological quality of a study. A high score on the PEDro-P scale only indicates that the internal validity of the study is not compromised, and the score should not be used to make judgments or decisions about clinical practice. Papers receiving a score of 5 or more on the PEDro scale are considered to be of moderate to high methodological quality [16]. To become familiar in using the PEDro-P scale, both the authors attended a free online training session that was hosted on a speech-language pathology practice database website called "speechBITE" (http:/ / www. speechbite.com/training/?page_id=2), which trained researchers to evaluate RCTs and NRCTs using the PEDro-P scale. After the training session, both the authors independently evaluated each of the five articles using the PEDro-P scale. Each study earned a point for a specific criterion on the scale only if it presented clear evidence regarding that criterion. After evaluating the five articles, both the authors compared the cumulative scores of each of the five articles. There were disagreements with regard to the methodological quality assessment of 2-3 criteria on the PEDro$\mathrm{P}$ scale in each study. These disagreements were clearly discussed and resolved, and a final score was assigned to each of the five articles with complete consensus.

The research design and evidence level of each of the five studies was determined based on the published guidelines of the American Speech-Language-Hearing Association [17]. These evidence levels are categorized as: level Ia for well-designed meta-analysis of $>1$ randomized controlled trial; level Ib for well-designed randomized controlled study; level IIa for well-designed controlled study without randomization; level IIb for well-designed quasiexperimental study; level III for well-designed non-experimental studies, including correlation and case studies; and level IV for expert committee report, consensus conference, clinical experience of respected authorities. Again, both the authors independently assigned an evidence level for each of the five studies. Disagreements $(n=2)$ were discussed and resolved with complete consensus.

\section{Results}

Of the 5 articles included in the current review, two articles employed parallel-group RCTs, one article used a retrospective design, one article used one-shot pre-post design, and finally one article employed a cross-over RCT design. The details of the five studies are discussed below. Specifically, information on nature of treatment, participants recruited, variables of treatment intensity that were manipulated, strengths and weaknesses, evidence level, and research design employed for each of the five studies are presented.

\section{Study 1}

McCullough et al. [10] investigated the outcomes of an intensive treatment regime of Mendelsohn maneuver in 18 post-stroke patients. The Mendelsohn maneuver is a swallowing maneuver used specifically to treat patients with reduced laryngeal excursion and limited cricopharyngeal opening. This maneuver requires the patient to hold his/her larynx in an elevated position (either using the hand or neck muscles) for an extended period of time during a voluntary swallow [18]. Eighteen post-stroke patients diagnosed with dysphagia who were recruited through convenience sampling participated in this study. The inclusion criteria for the participants were each participant had to be on some form of restricted diet and had to demonstrate at least a minimum amount of swallow with some material passing through the upper esophageal sphincter (UES). The onset of stroke for each patient ranged from 6 weeks to 22 months post-stroke ( $M=9.5$ months) at the time of participation. The baseline swallowing function of each patient was assessed through videofluoroscopic swallowing studies (VFSS). Each patient was randomly assigned to one of the two treatment groups. Group A received two weeks of treatment followed by two weeks of no treatment (BBAA). This arrangement was reversed in case of group $\mathrm{B}$ (AABB). 
During the treatment weeks, the first day was spent demonstrating and teaching the Mendelsohn maneuver to the patients by making use of surface electromyography feedback. Thus, the dose form was Mendelsohn maneuver. The actual treatment started from the second day. The patients were seen twice a day, with each session lasting between 45-60 min with a 2-3 hour break between the two sessions. The treatment dose on each day was $60-80$ swallows (depending on the participants' ability). The dose frequency during the first week of treatment was 8 sessions (as the first day was used for demonstration of the Mendelsohn maneuver), but the dose frequency during the second week was 10 sessions. The total intervention duration was 2 weeks. So the cumulative intervention ranged from 540-720 swallows (depending on the participants' ability) using Mendelsohn maneuver. VFSSs were conducted at the end of each week through the end of 4-week period. To assess the effect of Mendelsohn maneuver on hyolaryngeal excursion, the primary outcome measures were: (1) duration of hyoid maximum anterior excursion" (DOHMAE), (2) duration of hyoid maximum elevation (DOHME), and (3) duration of UES opening (DOUESO). The secondary duration measures were pharyngeal response duration, oral transit duration, pharyngeal transit duration, and standard duration. In addition, the quality of each swallow was assessed using standardized swallowing scales (e.g., penetration-aspiration scale). The principal investigator analyzed the data from tapes and DVDs that recorded the VFSS. The names of all the participants on the tapes and DVDs were replaced with deidentifying numbers that corresponded to participants. This ensured the blinding of the assessor to participant's identity.

The authors compared the mean values of each of the three duration measures (i.e. DOHME, DOHMAE, DOUESO) at the baseline phase to one-week post-treatment, two-weeks posttreatment, one-week of no-treatment, and two-weeks of notreatment using ' $P$ ' test for independent groups. The authors also analyzed the combined duration measure values of both the treatment groups (groups A and B) at the end of treatment weeks as well as no-treatment weeks. The results revealed that DOHME and DOHMAE values were significantly different at the end of 2 -weeks treatment phase in comparison to the baseline. However, these measures were not statistically different between the baseline and at the end of no-treatment phase. With regard to DOUESO, there were no statistical significant differences between the baseline phase and at the end of the treatment phase as well at the end of no-treatment phase. The authors just reported the mean values of all the other measures at baseline, end of no-treatment, and at the end of treatment phases. The descriptive results did not reveal significant improvement in swallowing physiology. In fact some measures got worse at the end of no-treatment phase, suggesting that there was no maintenance of treatment effects.

This study was a preliminary investigation of the effects of treatment intensity of Mendelsohn maneuver in improving swallowing physiology in post-stroke. Even though the findings of this study suggest that 540-720 swallows using Mendelsohn maneuver can improve hyolaryngeal excursion, it remains to be investigated if there are maintenance effects from this treatment regime. The findings of this study are confounded by several external variables such as heterogeneity of participants, lack of treatment fidelity, and lack of use of inferential statistical measures. Another disadvantage concerned the nature of one of the analyses. As mentioned before, the authors compared the baseline measures to one-week post-treatment, two-week post-treatment, one-week of no-treatment, and two-weeks of no-treatment. As the no- treatment weeks occurred after treatment weeks in about half the cases, the findings of the no-treatment weeks could have been influenced by the prior two weeks of treatment.Considering the variables that confounded the internal validity, this study obtained a "2" on the PEDro-P scale. As this study employed a randomized-controlled trial using crossover design, it was assigned to have level Ib evidence.

\section{Study 2}

Huckabee \& Cannito [19] retrospectively investigated the physiological and functional outcomes of an intense treatment program in 10 patients with chronic dysphagia subsequent to brainstem injury. Data for this retrospective study was collected from previous medical records, VFSS, and a patient questionnaire. The mean onset of dysphagia among the patients at the beginning of treatment was 26.9 months and ranged from 8-84 months. The treatment dose form for all the 10 patients was "Outpatient Accelerated Swallowing Treatment Program (OASTP)", which is an intense treatment program developed for patients with chronic dysphagia who had limited success with previous treatment(s). OATSP involves a combination of rehabilitative maneuvers such as modified Valsalva, the Mendelsohn, Masako, and head-lifting maneuvers that are used as deemed appropriate for the physiological needs of each patient. In addition to the maneuvers, the OASTP utilizes surface electromyography as a biofeedback tool and is paired with a rigorous home training program. The dose frequency of OASTP includes 2-sessions of 1-hr duration each provided every day for five consecutive days. There is a rest period of 2-4 hours between the 2 -sessions. The total duration of OASTP is 1 -week, thus amounting to 10 sessions within a 1 -week period. The home training component requires patients to practice the maneuvers for three additional sessions of 15 min duration during the 1 -week period. The cumulative intervention could not be determined, as the authors did not provide information on dose of OASTP.

The outcome measures were VFSS and the functional evaluation of oral intake. The VFSS were obtained on all the 10 patients pre-treatment and immediately post-treatment. The VFSS were evaluated using a 9-point rating scale, where " 0 " represented no significant dysphagia and "8" represented profound dysphagia. The functional outcome of oral intake was assessed by making use of a 5-point rating scale, where " 1 " represented feeding tube only and " 5 " represented oral intake only. The functional evaluation was carried out pre-treatment, immediate post-treatment, at a 6-month follow-up period, and at the conclusion of the study. The time period of obtaining the final functional evaluation rating varied from 1-4 years among the patients. The pre-treatment evaluation revealed that all the 10 patients demonstrated physiologic as well as functional evidence of dysphagia, with all the patients fed via gastrostomy or jejunostomy. The VFSS results at the end of 1-week treatment period revealed that there was a considerable change in the swallowing physiology in nine out of 10 patients. The functional evaluation results at the end of treatment period, at 6-month follow-up and at the termination of the study revealed that except for two patients, the remaining eight patients responded favorably to intervention by returning to complete oral intake. The statistical analyses also revealed a significant post-treatment effect on VFSS as well as on the functional evaluation of oral intake.

This study employed a retrospective design. A major limitation 
of retrospective design is that it cannot actively manipulate variables and observe its subsequent effects [20]. This study did not provide important information regarding who administered the treatment, blinding of the therapist(s), if the assessors were blinded to the study, and reliability of the outcome measures. While this study reports findings that are statistical significant, it does not report information regarding practical significance (i.e. effect sizes). Without knowing the effect size, it is difficult to estimate the magnitude of treatment effect [20]. All the above variables reduced the external as well as internal validity of this study, so it was awarded a "2" on the PEDro-P scale. Due to the retrospective design, this study was considered to have level III evidence.

\section{Study 3}

Bakhtiyari et al. [11] studied the effects of early swallowing intervention in 60 patients with dysphagia subsequent to stroke using a prospective single blind RCT design. The authors initially recruited 451 acute stroke patients, but had to exclude several patients due to multiple reasons, and finally 60 patients participated in the study. All the 60 patients were randomly and equally allotted to one of the three treatment groups based on the time of initiation of swallowing intervention. The three treatment groups were: (1) early initiation group (three days post-stroke), (2) medium group (two weeks post-stroke), and (3) late group (one month post-stroke). The dose form was traditional behavioral swallowing intervention that included a combination of compensatory and rehabilitative approaches. The compensatory approaches included enteral feeding by means of a nasogastric or percutaneous gastrostomy tube, modifying the food consistency, reducing the eating rate, postural correction to facilitate bolus transition, and maintaining adequate oral hygiene. The rehabilitative approaches involved airway-protection maneuvers, oral-motor exercises, thermal-tactile stimulation, and Shaker exercise. The authors did not provide information on dose. However, the dose frequency was $3 \times$ week. The total intervention duration was 3 months. $\mathrm{Cu}-$ mulative intervention could not be determined as there was no information on treatment dose. The outcome measures were: (1) scores obtained in the Northwestern dysphagia patient check sheet (NWDPCS), (2) VFSS, (3) scores on functional oral intake (FOI) scale, (3) frequency of pneumonia, and (4) number of intervention sessions required for improvement in swallowing. The post-treatment results of NWDPCS and VFSS revealed that patients receiving early intervention (early initiation group) demonstrated significant recovery over the other two groups. However, there were no differences between the medium and late groups. The mean number of sessions required for recovery of swallowing function in early, medium, and late groups were 10.25, 17.40, and 32.3 sessions, respectively. The statistical analysis revealed that the number of sessions required for recovery of swallowing function in early initiation group was significantly lower (10.25 \pm 1.91) than medium $(17.40 \pm 2.60)$ and late $(32.3 \pm 3.2)$ groups. However, there was no information if there was significant difference between the medium and late groups.

Although this study employed RCT design, there was lot of nuisance variables that confounded the findings. The intervention groups were not similar at baseline with regard to the frequency of pneumonia. The authors consider frequency of pneumonia as one of the outcome measures, but it is surprising that none of the patients in the early initiation group had pneumonia. There is no information regarding the blinding of therapists and blinding of assessors, which can potentially confound the internal validity of the study. There is also no information on treatment fidelity. The authors considered the scores on FOI scale as one of the outcome measures, but did not report any findings related to it. This makes us question the necessity of including scores on FOI scale as one of the outcome measures. Finally, the authors do not report effect sizes of the three treatment groups, making it difficult to estimate the magnitude of treatment effect between the three groups. As the study used a parallel-group RCT, it was assigned Ib level evidence. It scored " 5 " on the PEDro-P scale suggesting moderate methodological quality.

\section{Study 4}

El Sharkawi et al. [21] investigated the effects of Lee Silverman Voice Treatment (LSVT) on swallowing and voice in eight patients diagnosed with idiopathic Parkinson's disease (PD). The age of the participants ranged from 57-77 years and mean age was 69.8 years. There was no information reported about the onset of PD for the participants. LSVT is an intense treatment program based on principles of motor learning and is designed for treating speech and voice deficits associated with PD [21]. The dose form of LSVT is sensory retraining and instructions encouraging the patients to think loudly. The exact dose of LSVT is often not reported $[22,23]$. The dose frequency of a typical LSVT program is 4-times per week. The duration of each individual session is around 50 -to-60 min. The total duration of LSVT is 4-weeks. In addition to receiving LSVT, patients also complete 5-to-10 min of homework exercises on treatment days and 20-to-30 min on non-treatment days [22]. It is difficult to determine the cumulative intervention of LSVT due to unavailability of data on dose. The authors of this study investigated the effects of LSVT using a one-shot pretest-posttest design. The voice and swallowing function of all the patients who participated in the study were evaluated pre as well as post-treatment. The swallowing evaluation included motility and temporal measures that were assessed using VFSS during consumption of different boluses. The motility measures were assessed by having the assessors review the videotape of each swallow in slow motion. Assessment of temporal measures involved making note of several parameters such as oral transit time, pharyngeal transit time, pharyngeal delay time, and so forth. A global swallow measure referred to as the "oropharyngeal swallow efficiency" measure was determined through a formula that included various motility and temporal parameters. Assessment of voice involved measuring the sound pressure level (SPL) during reading and sustained phonation as well as measuring the mean fundamental frequency (F0) during reading. Patients were also asked to self-evaluate their voices before and after treatment using visual analogue scale and voice handicap index.

Comparison of pre and post-treatment results with regard to swallowing revealed that even though several motility measures improved, functional swallow continued to be affected (and even got worse) post-treatment across different boluses in many patients. The authors reported the improvement in motility parameters using descriptive statistics. The results of temporal measures revealed that there was a significant improvement of oral transit time post-treatment only during consumption of $3 \mathrm{ml}$ liquid bolus and pudding (paste). The oral residue percentage significantly improved post-treatment only during consumption of 3 and 5 $\mathrm{ml}$ liquid boluses. Finally, the oropharyngeal swallow efficiency improved post-treatment only during drinking of barium liquid from a cup. There were no other significant improvements. With regard to the voice, comparison of pre and post-treatment results 
revealed that there was significant improvement of SPL during sustained phonation as well as reading tasks. The F0 also significantly improved during the reading tasks. However, there was no improvement in patients' self-rating of their voices.

The effects of LSVT on improving vocal function in patients with PD are well known [22]. This was a pilot study to investigate if the beneficial effects of LSVT can be extended to swallowing as well. The authors minimized the influence of external variables by blinding the assessors to the nature of data, excluding the therapists from assessment and there was no patient attrition. However, the authors did not perform reliability of the assessed data and did not provide information on treatment effect size. In addition, results revealed that there was improvement in only certain swallowing outcome measures. Based on these findings, the beneficial effects of LSVT on improving swallowing function in individuals with PD can be only speculated. The nature of research design and a small number of participants limit the generalization of the results of this study. As this study used a one-shot pre-post design without a control group, it was assigned IIb evidence level. The study was awarded a score " 2 " on the PEDro-P scale suggesting poor methodological quality as it failed to control variables that confounded the internal validity.

\section{Study 5}

Carnaby, Hankey, and Pizzi [24] investigated the effect of treatment intensity on recovery of swallowing function in patients post-stroke. A total of 3,227 patients with suspected clinical stroke were screened for inclusion in the study. The inclusion criteria were: (1) a diagnosis of stroke within seven days postonset by the attending clinician, (2) clinical diagnosis of dysphagia by the study speech-language pathologist, (3) no prior history of swallowing treatment, and (4) no prior surgery of the head or neck. Several patients has to be excluded as they failed to meet the inclusion criteria. Finally, 306 patients met the inclusion criteria. These 306 patients were equally and randomly allotted to one of the three treatment groups: usual care group, high-intensity group, and low-intensity group. The patients and the study speech-language pathologist were not concealed to the treatment allocation. In usual care group, patients received intervention from attending physicians as per usual practice. Physicians referred patients to the hospital speech-language pathologist(s) only if it was deemed necessary. If these patients received intervention, the dose form was supervision and precautions for safe feeding and swallowing (e.g., slowed rate of feeding). Due to the inconsistent nature of intervention received by the patients, dose and dose frequency could not be determined. The dose form for the low-intensity group was environmental modifications for feeding and swallowing such as upright positioning for feeding, safe swallowing advice, and appropriate dietary modification under the direction of the study speech-language pathologist. The dose frequency was $3 \times$ week, the total treatment duration was 1 -month (or less if the patients were discharged earlier than that). In high-intensity group, the dose form consisted of direct swallowing exercises such as effortful swallowing and supraglottic swallow technique and appropriate dietary modifications under the supervision of the study speech-language pathologist. Treatment was provided every working for a month (or less if the hospital stay was less than a month). Considering that the treatment services were provided for 5-days a week (excluding the weekends), the dose frequency was estimated to be $5 \times$ week, and total duration was estimated to be 4 -weeks. As dose was not reported for low as well as high-intensity treatment groups, cumulative intervention could not be determined for both the groups.

The primary treatment outcome measure was the proportion of the patients who returned to their normal pre-stroke diet within 6 months after initiation of treatment. The secondary outcome measures include the time duration for patients to return to their normal diet, proportion of patients who had achieved functional swallowing or developed one or more swallowing complications, and the proportion of patients who had died or were institutionalized, or dependent to carry out activities of daily living. The follow-up results at 6-months post-discharge was collapsed for both high as well as low-intensity treatment groups (referred to as the standard care group) and was compared with the outcomes of the usual care group. The results revealed that patients receiving standard care demonstrated significant improvement in achieving functional swallowing and lesser proportion of patients exhibited swallowing complications in comparison to patients receiving usual care. However, the outcome measures related to death, institutionalization, and dependency for activities of daily living did not vary as a function of intensity of swallowing treatment.

This study used a RCT design, thereby minimizing the influence of nuisance variables. The study followed "intend to treat" approach, blinded the assessor to treatment allocation, recruited a large sample size thereby increasing the power of the study and controlling Type II error probability. However, some of the major drawbacks were, the study speech-language pathologist as well as the patients were not blinded to treatment allocation, the study was restricted to a single geographical region in Australia which limits the generalization of the results, the authors reported the combined data of high as well as low-treatment intensity groups, which masks valuable information related to effects of high and low treatment intensity, and finally, the treatment dose was not controlled across the three treatment groups. Even though, the duration of treatment received by standard treatment group was more than the usual care group, the usual care group received treatment on more number of days than the standard treatment group, which could have confounded the effect of treatment dose on outcome measures. On the PEDro-P scale, this study received the highest score " 6 " in comparison to the other four studies. As it employed a parallel group RCT design, this study was considered to demonstrate Ib level evidence. The details of methodological quality analysis and evidence level for the five studies included in the review are presented in Table 1. The best available evidence for behavioral treatment intensity for dysphagia with regard to each variable of treatment intensity is summarized in Table 2.

\section{Discussion}

The issue of treatment intensity has been one of the most debated topics in speech-language pathology. An appropriate solution to this ongoing debate is to review the existing evidence for treatment intensity across a range of speech, language, and swallowing disorders, and to identify the potential for future research. Although determining optimal treatment intensity is a challenging line of research in speech-language pathology, it begs further investigation. To our knowledge, this is the first study to review evidence for optimal behavioral treatment intensity for dysphagia. The five studies that were included in this review differed from one another with regard to research design, evidence level and methodological quality. It is important to be aware that methodo- 
Table 1. Details of methodological quality analysis using PEDro-P scale and evidence level for the five studies included in the review.

\begin{tabular}{|c|c|c|c|c|c|}
\hline Criteria/Study & $\begin{array}{l}\text { McCullough } \\
\text { et al. (2012) }\end{array}$ & $\begin{array}{c}\text { Huckabee \& } \\
\text { Cannito (1999) }\end{array}$ & $\begin{array}{l}\text { Bakhtiyari et } \\
\text { al. (2015) } \\
\end{array}$ & $\begin{array}{l}\text { El Sharkawi } \\
\text { et al. (2002) }\end{array}$ & $\begin{array}{c}\text { Canaby } \\
\text { et al (2006) }\end{array}$ \\
\hline Eligibility criteria were specified & Yes & Yes & Yes & No & Yes \\
\hline $\begin{array}{l}\text { Subjects were randomly allocated to } \\
\text { interventions (inacrossover study, } \\
\text { subjects were randomly allocated } \\
\text { an order in which treatments were } \\
\text { received) } \\
\end{array}$ & Yes & No & Yes & No & Yes \\
\hline Allocation was concealed & No & No & No & No & Yes \\
\hline $\begin{array}{l}\text { The intervention groups were simi- } \\
\text { lar at baseline regarding the most } \\
\text { important prognostic indicators }\end{array}$ & No & No & Yes & No & No \\
\hline There was blinding of all subjects & No & No & Yes & No & No \\
\hline $\begin{array}{c}\text { There was blinding of all therapists } \\
\text { who administered the therapy }\end{array}$ & No & No & No & No & No \\
\hline $\begin{array}{c}\text { There was blinding of all asses- } \\
\text { sors who measured atleast one key } \\
\text { outcome }\end{array}$ & Yes & No & No & No & Yes \\
\hline $\begin{array}{c}\text { Measures of at least one key } \\
\text { outcome were obtained from more } \\
\text { than } 85 \% \text { of the subjects initially } \\
\text { allocated to groups }\end{array}$ & No & Yes & No & Yes & No \\
\hline $\begin{array}{l}\text { All subjects for whom outcome } \\
\text { measures were available received } \\
\text { the treatment or control condition } \\
\text { as allocated or, where this was not } \\
\text { the case, data for at least one key- } \\
\text { outcome was analysed by "inten- } \\
\text { tion to treat" }\end{array}$ & No & No & No & No & Yes \\
\hline $\begin{array}{l}\text { The results of between- interven- } \\
\text { tion group statistical comparisons } \\
\text { are reported for at least one key- } \\
\text { outcome }\end{array}$ & No & No & Yes & No & Yes \\
\hline $\begin{array}{l}\text { The study provides both point } \\
\text { measures and measures of variabil- } \\
\text { ity for at least one keyoutcome }\end{array}$ & No & Yes & Yes & Yes & Yes \\
\hline $\begin{array}{c}\text { Total score on the PEDro-P scale } \\
\text { out of } 10 \text { (after consensus) }\end{array}$ & 2 & 2 & 5 & 2 & 6 \\
\hline Evidence level (after consensus) & $\mathrm{Ib}$ & III & $\mathrm{Ib}$ & $\mathrm{IIb}$ & $\mathrm{Ib}$ \\
\hline
\end{tabular}

logical quality and evidence level are only indicators of external and internal validity of a study, they cannot determine the benefits of a treatment program. For example, it is possible that a certain treatment program has positive outcomes in spite of having minimal scientific evidence.

Of the five studies, only one study by McCullough et al. [10] presented sufficient information that allowed us to determine the cumulative treatment intensity. Thus, the existing evidence suggests that a cumulative treatment intensity of 540-720 swallows using the Mendelsohn maneuver seems to be beneficial for patients with pharyngeal dysphagia subsequent to a brainstem stroke. But again, this finding should be interpreted with caution. Even though, McCullough et al. used a randomized crossover design to conduct this study, there were several nuisance variables that could have confounded the findings. A major limitation of this study was the approach used by the authors to analyze the data.
The authors analyzed the data by combining the treatment-no treatment as well the no treatment-treatment groups. The carry over effects from the treatment phase to the no-treatment phase and vice-versa could have influenced the findings to a significant extent. Even though, the evidence level for all the studies (except Huckabee \& Cannito [19] -level III evidence) included in this review seemed to be commendable, the findings do not seem to benefit clinical practice in dysphagia due to unavailability of information on treatment dose, which in turn did not allow us to determine cumulative treatment intensity. The question "how much therapy is sufficient" can be answered effectively only if studies report information on all aspects of the treatment intensity including treatment dose.

There were also several other key aspects that the reviewed studies did not account for. All the studies failed to report effect sizes. When comparing a treatment and a control group or a pre vs. 
Table 2. Best available evidence from each of the five studies with regard to behavioral treatment intensity for dysphagia.

\begin{tabular}{|c|c|c|c|c|c|}
\hline $\begin{array}{c}\text { Treatment } \\
\text { variable/Study }\end{array}$ & $\begin{array}{l}\text { McCullough } \\
\text { et al. (2012) }\end{array}$ & $\begin{array}{l}\text { Huckabee \& } \\
\text { Cannito (1999) }\end{array}$ & $\begin{array}{l}\text { Bakhtiyari } \\
\text { et al. (2015) }\end{array}$ & $\begin{array}{l}\text { El Sharkawi } \\
\text { et al. (2002) }\end{array}$ & $\begin{array}{c}\text { Carnaby et } \\
\text { al. (2006) }\end{array}$ \\
\hline Dose form & $\begin{array}{l}\text { Mendelsohn } \\
\text { maneuver + } \\
\text { SEMG feed- } \\
\text { back }\end{array}$ & OASTP & $\begin{array}{l}\text { Traditional } \\
\text { behavioral } \\
\text { swallowing } \\
\text { intervention }\end{array}$ & $\begin{array}{c}\text { LSVT - } \\
\text { sensory } \\
\text { retraining } \\
\text { and simple } \\
\text { instructions }\end{array}$ & $\begin{array}{c}\text { Swallowing } \\
\text { exercises }\end{array}$ \\
\hline Dose & 60-80 swallows & Not reported & Not reported & Not reported & Not reported \\
\hline Dose frequency & $\begin{array}{c}8 \times 1^{\text {st }} \text { week } \\
10 \times 2^{\text {nd }} \text { week } \\
(45-60 \text { min ses- } \\
\text { sions })\end{array}$ & $\begin{array}{c}10 \times \text { week }(1-\mathrm{hr} \\
\text { sessions }) \\
3 \times \text { week }(15- \\
\text { min duration } \\
\text { sessions at } \\
\text { home })\end{array}$ & $3 \times$ week & $\begin{array}{c}\times \text { week } \\
(50-60 \mathrm{~min} \\
\text { sessions })\end{array}$ & $\begin{array}{c}5 \times \text { week (1- } \\
\text { hr/day) }\end{array}$ \\
\hline $\begin{array}{l}\text { Total interven- } \\
\text { tion duration }\end{array}$ & 2 weeks & 1 week & 3 months & 4 weeks & 1 month \\
\hline $\begin{array}{c}\text { Cumulative } \\
\text { intervention } \\
\text { intensity }\end{array}$ & $\begin{array}{l}540-720 \text { swal- } \\
\text { lows (based on } \\
\text { participant's } \\
\text { ability) }\end{array}$ & CND & CND & CND & CND \\
\hline
\end{tabular}

$\mathrm{CND}=$ could not be determined; OASTP $=$ Outpatient Accelerated Swallowing Treatment Program

post-treatment conditions, it is important to report the statistical significance between the groups and/or conditions, but it is also equally important to report data on practical significance. This helps us to determine the magnitude of differences between the groups and/or conditions, if at all there is a significance difference between them [25]. One of the ideal measures to estimate practical significance is effect size [26,27]. So in all the studies, we know that there were significant changes between the treatment and control groups or between the pre and post-treatment conditions, but we do not know the magnitude of these differences, as the effect sizes were not reported. The next aspect that the studies failed to consider was reporting the maintenance data. Except Carnaby et al. [24] and Huckabee and Cannito [19], all the other studies measured treatment outcomes immediately at the end of treatment sessions. While this a logical approach to document the changes subsequent to treatment, researchers should also consider reporting maintenance data. As patients with dysphagia are relearning their lost skills, it is crucial to examine if the learned behavior post-treatment is maintained across a certain time period. In accordance with the motor learning literature, if patients with dysphagia demonstrate long-term maintenance of swallow function post-treatment, then that is an indicator of a permanent change in their behavior to swallow safely and effectively [28]. Finally, the methodological quality of five the studies ranged from poor to moderate, questioning the validity of the findings in these studies. Future research should consider addressing these limitations so that we are able to determine optimal treatment intensity across different severity levels of dysphagia.

\section{Conclusion}

Although the current review reveals some interesting findings, it is obviously not without limitations. First, the current review included studies that recruited only patients with dysphagia subsequent to a stroke. Moreover, the patient population across the five studies were heterogeneous in regard to the age of onset of dysphagia. Previous research indicates that the treatment intensity for head and neck cancer patients with chemotherapy and/or radiation will be different than for acute stroke patients which will be different than chronic stroke patients [29, 30]. Unfortunately, the search process did not yield any studies that investigated treatment intensity for dysphagia in patients with head and neck cancer receiving chemotherapy and/or radiation. So for this reason, the findings of this review need to be interpreted in the context of the studies that were examined.

Second, factors such as nature of dysphagia intervention, therapy goals that are to be achieved, and neural/structural changes that occur over the course of intervention could serve as external variables preventing the generalization of the current findings. Prior to the initiation of therapy, it is important for a dysphagia clinician to be aware of the goals that his/her clients would like to achieve [31]. Prior research in exercise science suggests that the nature of intervention should differ based on whether the goal is to promote speed, strength, endurance, or a combination of these [32]. During the strength training regime, early changes reflect the modifications in the way nervous system activates the muscles rather than the structural changes in the muscle itself. However, as the training progresses, changes appear more to be at the level of structural alterations to the muscle because of the decrease in the contribution of the nervous system. So it is likely that the treatment intensity also differs in response to the neural and structural changes over the course of intervention [31]. As all the above three factors can have a bearing on treatment intensity in dysphagia, the findings of the current review should be extrapolated with caution.

Finally, the assessors (both the authors) were not blinded to information such as the names of the authors and the published dates of the articles that were reviewed, which could introduce a source of bias. In spite of these limitations, the current study systematically reviewed as well as evaluated the rigor of some of the 
treatment studies that met the inclusion criteria using appropriate appraisal tools. The results suggest that there is limited evidence regarding behavioral treatment intensity for dysphagia, and there is a need for well-controlled group design studies to expand on this line of research.

\section{Appendix}

\section{References}

[1]. Bhattacharyya $\mathrm{N}$ (2014) The prevalence of dysphagia among adults in the United States. Otolaryngol Head Neck Surgery 151(5): 765-769.

[2]. Sura L, Madhavan A, Carnaby G, Crary MA (2012) Dysphagia in the elderly: management and nutritional considerations. Clin Interv Aging 7: 287-298.

[3]. Cichero JAY, AltmanCJ (2012) Stepping Stones to Living Well with Dysphagia. Nestlé Nutrition Institute Workshop Series 72: 1-11.

[4]. Swallowing Disorders (Dysphagia) in Adults, American Speech-Language-Hearing Association, 2015,Accessed on September 13, 2015 from http://www.asha.org/public/speech/swallowing/Swallowing-Disorders-inAdults/\#dx

[5]. Logemann JA (2012) Clinical efficacy and randomized clinical trials in dysphagia. Int J Speech Lang Pathol 14(5): 443-446.

[6]. Baker E (2012) Optimal intervention intensity. Int J Speech Lang Pathol 14(5): 401-409.

[7]. Bhogal SK, Teasell R, Speechley M (2003) Intensity of aphasia therapy, impact on recovery. Stroke 34(4): 987-993.

[8]. Brandel J, Loeb DF (2011) Program intensity and service delivery models in the schools: SLP survey results. Lang Speech Hear Serv Sch 42(4): 461-490.

[9]. Warren SF, Fey ME, Yoder PJ (2007) Differential treatment intensity research: A missing link to creating optimally effective communication interventions. Ment Retard Dev Disabil Res Rev 13(1): 70-77.

[10]. McCullough GH, Kamarunas E, Mann GC, Schmidley JW, Robbins JA, et al. (2012) Effects of Mendelsohn maneuver on measures of swallowing duration post stroke. Top Stroke Rehabil 19(3): 234-243.

[11]. Bakhtiyari J, Sarraf P, Nakhostin-Ansari N, Tafakhori A, Logemann J, et al (2015) Effects of early intervention of swallowing therapy on recovery from dysphagia following stroke. Iran J Neurol 14(3): 119-124.

[12]. Thomas RM, Kaipa R (2015) The use of non-speech oral-motor exercises among Indian speech-language pathologists to treat speech disorders: An online survey. S Afr J Commun Disord 62(1): E1-E12.

[13]. Liberati A, Altman DG, Tetzlaff J, Mulrow C, Gøtzsche PC, et al (2009) The PRISMA statement for reporting systematic reviews and meta-analyses of studies that evaluate health care interventions: explanation and elaboration. Ann Intern Med 151(4): W-65-W-94.

[14]. Murray E, Power E, Togher L, McCabe P, Munro N, et al. (2013) The reliability of methodological ratings for speechBITE using the PEDro-P scale.
Int J Lang Commun Disord 48(3): 297-306.

[15]. Sherrington C, Herbert RD, Maher CG, Moseley AM (2000) PEDro. A database of randomized trials and systematic reviews in physiotherapy. Man Ther 5(4): 223-226.

[16]. Moseley A, Sherrington C, Herbert R, Maher C (2000) The extent and quality of evidence in neurological physiotherapy: an analysis of the Physiotherapy Evidence Database (PEDro). Brain Impair 1(2): 130-140.

[17]. Evidence-based practice in communication disorders: An introduction (2004) [Technical report], American Speech-Language-Hearing Association. http://www.asha.org/policy/tr2004-00001/

[18]. Lazarus C, Logemann JA, Gibbons P (1993) Effects of maneuvers on swallowing function in a dysphagic oral cancer patient. Head Neck 15(5): 419424.

[19]. Huckabee ML, Cannito MP (1999) Outcomes of swallowing rehabilitation in chronic brainstem dysphagia: a retrospective evaluation. Dysphagia 14(2): 93-109.

[20]. Dollaghan C (2007) The Handbook for Evidence-Based Practice in Communication Disorders. Paul H. Brooks Publishing Co, Baltimore, US.

[21]. El Sharkawi A, Ramig L, Logemann J, Pauloski BR, Rademaker A, et al. (2002) Swallowing and voice effects of Lee Silverman Voice Treatment (LSVT'): a pilot study. J Neurol Neurosurg Psychiatry 72(1): 31-36.

[22]. Ramig LO, Sapir S, Fox C, Countryman S (2001) Changes in vocal loudness following intensive voice treatment $\left(\mathrm{LSVT}^{\circ}\right)$ in individuals with Parkinson's disease: A comparison with untreated patients and normal age-matched controls. Mov Disord 16(1): 79-83.

[23]. Sapir S, Spielman JL, Ramig LO, Story BH, Fox C (2007) Effects of intensive voice treatment (the Lee Silverman Voice Treatment [LSVT]) on vowel articulation in dysarthric individuals with idiopathic Parkinson disease: acoustic and perceptual findings. J Speech Lang Hear Res 50(4): 899-912.

[24]. Carnaby G, Hankey GJ, Pizzi J (2006) Behavioural intervention for dysphagia in acute stroke: a randomised controlled trial. Lancet Neurol 5(1):31-37.

[25]. Bothe AK, Richardson JD (2011) Statistical, practical, clinical, and personal significance: Definitions and applications in speech-language pathology. Am J Speech Lang Pathol 20(3): 233-242.

[26]. Cohen J (1988) Statistical power analysis for the behavioral sciences. (2nd edtn), Erlbaum, Hillsdale, New Jersey.

[27]. Keppel G, Wickens TD (2004) Design and analysis: A researcher's handbook. (4th edtn), Pearson Prentice Hall, Upper Saddle River, New Jersey.

[28]. Sheppard JJ (2008) Using motor learning approaches for treating swallowing and feeding disorders: A review. Lang Speech Hear Serv Sch 39(2): 227-236.

[29]. Pauloski BR (2008) Rehabilitation of dysphagia following head and neck cancer. Phys Med Rehabil Clin N Am 19(4): 889-928.

[30]. Geeganage C, Beavan J, Ellender S, Bath PM (2012) Interventions for dysphagia and nutritional support in acute and subacute stroke. The Cochrane Library.

[31]. Burkhead LM, Sapienza CM, Rosenbek JC (2007) Strength-training exercise in dysphagia rehabilitation: principles, procedures, and directions for future research. Dysphagia 22(3): 251-265.

[32]. Deschenes M, Kraemer W (2002) Performance and physiologic adaptations to resistance training. Am J Phys Med Rehabil 81(11 Suppl): S3-S16. 